\title{
COMPORTAMENTO ELETROQUÍMICO DA N-NITROSOTIAZOLIDINA ÁCIDO CARBOXÍLICO SOBRE ELETRODOS DE OURO E MERCÚRIO
}

\author{
Lucia Codognoto \\ Instituto de Pesquisa e Desenvolvimento, Universidade do Vale do Paraíba, Av. Shishima Hifumi, 2911, 12244-000 São José dos \\ Campos - SP, Brasil
}

Felix G. R. Reyes

Departametno de Ciência de Alimentos, Faculdade de Engenharia de Alimentos, Universidade Estadual de Campinas, CP 6121, 13083-970 Campinas - SP, Brasil

\section{Eduardo Winter e Susanne Rath*}

Departamento de Química Analítica, Instituto de Química, Universidade Estadual de Campinas, CP 6154, 13084-971 Campinas - SP, Brasil

Recebido em 11/4/07; aceito em 17/12/07; publicado na web em 31/7/08

\begin{abstract}
ELECTROCHEMICAL BEHAVIOR OF N-NITROSOTHIAZOLIDINE CARBOXILIC ACID ON GOLD AND MERCURY ELECTRODES. The electrochemical behavior of N-nitrosothiazolidine carboxylic acid (NTAC) on gold and hanging mercury electrodes, using the cyclic and square wave voltammetries, was studied. Whereas NTAC suffer reduction in a single step on the mercury electrode, two peaks appears on the cyclic voltammograms on the gold electrode, one anodic peak overlaying the gold oxide process at $1.2 \mathrm{~V}$ and one cathodic peak at $-0.41 \mathrm{~V} v s \mathrm{Ag} / \mathrm{AgCl}, \mathrm{KCl} 3.0 \mathrm{~mol} \mathrm{~L}^{-1}$. The cathodic peak depends on the previous oxidation of NTAC at the electrode surface, presents irreversible and adsorption controlled characteristics and it is suitable for quantitative purposes.
\end{abstract}

Keywords: N-nitrosothiazolidine carboxylic acid; voltammetry; gold electrode.

\section{INTRODUÇÃO}

Os compostos $\mathrm{N}$-nitrosos, e em particular as N-nitrosaminas, são considerados potentes carcinógenos, além de apresentarem ação teratogênica e mutagênica em animais de laboratório. As nitrosaminas podem ser encontradas nas mais diversas matrizes, entre essas, alimentos, solos, água, ar, pesticidas, cigarros, produtos de borracha e cosméticos. Nos alimentos podem ser encontradas em diversos produtos, principalmente nos industrializados, como presunto, salsicha, salame e outros produtos cárneos, nos quais são formadas a partir de precursores nitrosáveis do próprio alimento e de agentes nitrosantes. ${ }^{1-3}$ É importante ressaltar que, devido ao nitrato poder ser reduzido a nitrito na cavidade bucal e, sendo a faixa ótima de $\mathrm{pH}$ para a formação de $\mathrm{N}$-nitrosaminas semelhante àquela encontrada no estômago do homem após ingestão de alimentos, a formação de N-nitrosaminas in vivo torna-se um assunto de extrema importância.,

Para fins analíticos, as $\mathrm{N}$-nitrosaminas são divididas em voláteis e não voláteis. As diferenças nas propriedades químicas e fisicoquímicas desses compostos dificultam o estabelecimento de métodos analíticos de aplicação geral. ${ }^{6}$ Todavia, os métodos cromatográficos têm sido os mais utilizados na determinação de nitrosaminas em matrizes diversas, onde a cromatografia líquida de alta eficiência e a cromatografia gasosa tem sido empregadas para a determinação das nitrosaminas não voláteis e voláteis, respectivamente. Os detectores mais utilizados em ambas as técnicas têm sido o de quimiluminescência (TEA - Thermal Energy Analyzer), uma vez que esse é seletivo para compostos que contêm o grupo N-nitroso ${ }^{7-9}$ e o de massas. ${ }^{10,11}$

Entretanto, as técnicas cromatográficas associadas ao detector de quimiluminescência ou ao espectrômetro de massa requerem alto investimento inicial e, em função disto, poucas indústrias no

*e-mail: raths@iqm.unicamp.br
Brasil controlam seus produtos quanto à presença de nitrosaminas. O Brasil tem inúmeros alimentos típicos, assim como situações diferenciadas de processamento de alimentos que propiciam a formação de N-nitrosaminas (processamento caseiro de bacon, elaboração e comercialização de lingüiças de preparo tipo caseiro). Desta forma, novos métodos de análises para a determinação de nitrosaminas são de grande interesse, principalmente se forem de menor custo, envolverem menor número de etapas no preparo de amostra e menor consumo de solventes. Nesta direção, as técnicas eletroanalíticas surgem como uma alternativa para a determinação de compostos orgânicos e inorgânicos em matrizes diversas.

A determinação eletroquímica de nitrosaminas tem sido realizada de maneira muito tímida, sendo que a maioria dos trabalhos citados na literatura trata da redução eletroquímica destes compostos sobre o eletrodo de mercúrio. Neste sentido, Goicolea et al. ${ }^{12}$ utilizaram um eletrodo de mercúrio como detector eletroquímico, associado à cromatografia líquida de alta eficiência, para a determinação de N-nitrosopiperidina (NPIP) em cervejas. A fase móvel utilizada foi metanol-acetonitrila-tampão fosfato $\mathrm{pH} 3,5$ (57:38:5 v/v/v) com o detector operando no modo de corrente direta (DC) a potencial de $-1250 \mathrm{mV}$. O limite de detecção obtido foi de $0,2 \mathrm{ng}$ com um desvio padrão relativo de $2,8 \%$.

Em um outro estudo, ${ }^{13}$ foi descrito um método para a determinação de dinitrosopiperazina (DNPZ) em suco gástrico artificial, utilizando a polarografia de pulso diferencial. A DNPZ apresentou um pico catódico em $-0,77 \mathrm{~V}$ vs $\mathrm{Ag} / \mathrm{AgCl}$, com processo controlado por difusão. O intervalo de concentração estudado foi de 0,4 a $24 \mu \mathrm{g}$ $\mathrm{mL}^{-1}$ e o limite de detecção foi de $0,072 \mu \mathrm{g} \mathrm{mL}^{-1}$.

No entanto, um dos principais problemas quanto a utilização do eletrodo de mercúrio (HMDE) para a determinação de nitrosaminas está associado ao fato do sinal analítico observado ser referente à redução do grupo $\mathrm{N}$-nitroso, característico a todas as nitrosaminas. Sendo assim, 
não há possibilidade da determinação seletiva das mesmas e apenas é possível a determinação de nitrosaminas totais.

Outros estudos encontrados na literatura tratam da determinação eletroanalítica de nitrosaminas utilizando eletrodos quimicamente modificados com polímeros orgânicos e inorgânicos. ${ }^{14-16} \mathrm{~A}$ utilização de voltametria adsortiva de redissolução sobre estes eletrodos pode atingir limites de detecção da ordem de $10^{-10} \mathrm{~mol} \mathrm{~L}^{-1}$ para diferentes nitrosaminas. ${ }^{17}$ No entanto, um dos problemas observados com a utilização destes materiais eletródicos é que ocorre adsorção das espécies na superfície inibindo a resposta e, conseqüentemente, ocorre uma diminuição da sensibilidade do método proposto. ${ }^{18}$

O objetivo deste trabalho foi realizar um estudo comparativo do comportamento eletroquímico da N-nitrosotiazolidina ácido carboxílico (NTAC), que é uma nitrosamina não volátil encontrada principalmente em produtos defumados, sobre os eletrodos de ouro e de mercúrio (HMDE), utilizando a voltametria de onda quadrada (SWV), visando assim desenvolver um método eletroanalítico para a determinação de NTAC.

\section{PARTE EXPERIMENTAL}

\section{Reagentes e soluções}

A solução estoque de NTAC foi preparada em meio aquoso, na concentração de $1,00 \times 10^{-3} \mathrm{~mol} \mathrm{~L}^{-1}$. As demais soluções foram preparadas a partir da solução estoque mediante diluição com água.

Como eletrólito suporte foi utilizada uma solução de fosfato de sódio na concentração de $0,10 \mathrm{~mol} \mathrm{~L}^{-1}$. $\mathrm{O}$ pH desta solução foi ajustado, conforme necessário, com $\mathrm{NaOH}$ 1,0 $\mathrm{mol} \mathrm{L}^{-1}$ ou com $\mathrm{H}_{3} \mathrm{PO}_{4} 1,0 \mathrm{~mol} \mathrm{~L}^{-1}$.

Todas as soluções foram preparadas com água purificada em sistema Milli-Q, procedência Millipore Corporation.

\section{Instrumentação}

A célula eletroquímica utilizada para as medidas com o eletrodo de ouro foi uma célula convencional de compartimento único com orifícios para o encaixe dos eletrodos. Os estudos foram realizados utilizando um eletrodo de ouro $(\phi=1 \mathrm{~mm})$ como eletrodo de trabalho, um eletrodo de $\mathrm{Ag} / \mathrm{AgCl}$ em $\mathrm{KCl}$ 3,0 mol L-1 como referência e um fio de platina como eletrodo auxiliar.

As medidas com o eletrodo de mercúrio (HMDE) foram realizadas em um Sistema Stand 663 VA (Metrohm) contendo um eletrodo de trabalho de gota pendente de mercúrio (área: $0,52 \mathrm{~mm}^{2}$ ), um eletrodo de referência $\mathrm{Ag} / \mathrm{AgCl}, \mathrm{KCl} 3,0 \mathrm{~mol} \mathrm{~L}^{-1}$ e um eletrodo auxiliar de grafite pirolítico.

Para as medidas eletroquímicas foi utilizado o potenciostato/galvanostato Autolab ${ }^{\circledR}$ PGSTAT 30 (Eco Chemie). A aquisição dos dados e o gerenciamento do potenciostato foram realizados por um microcomputador (Dell) e mediante programa computacional GPES.

Todas as soluções tiveram seus valores de $\mathrm{pH}$ ajustados utilizando um pH mêtro da Digimed DM-20 (Brasil) e um eletrodo combinado de vidro também da Digimed - Instrumentação analítica. O pHmêtro era diariamente calibrado com soluções tampão comerciais de pH 4 e 7.

\section{RESULTADOS E DISCUSSÃO}

A NTAC foi o composto escolhido para este estudo por ser representante do grupo das nitrosaminas não voláteis e também porque não foram encontrados registros na literatura sobre o comportamento eletroquímico desta nitrosamina. O comportamento eletroquímico da NTAC foi estudado sobre os eletrodos de ouro e HMDE.

O eletrodo HMDE foi também utilizado nos estudos, visto que dados da literatura indicam que outras nitrosaminas sofrem redução sobre esta superfície e o sinal observado está relacionado com a redução do grupo N-nitroso, presente em todas as nitrosaminas. Assim, os resultados obtidos com o eletrodo HMDE foram utilizados como parâmetro de comparação para os dados encontrados para a NTAC sobre o eletrodo de ouro.

\section{Estudo do comportamento eletroquímico de NTAC sobre o eletrodo de ouro}

Resposta voltamétrica da NTAC sobre o eletrodo de ouro

$\mathrm{O}$ voltamograma da NTAC, no intervalo de $-0,60$ a 1,55 V, apresenta um pico anódico (Ia) em torno de $1,20 \mathrm{~V}$ (região da formação dos óxidos de ouro) e um pico catódico (Ic) em -0,45 V (Figura 1).

Foi verificado que os picos Ia e Ic são dependentes entre si e que as intensidades de corrente aumentam proporcionalmente com a concentração de NTAC na célula. Adicionalmente, observou-se que a corrente catódica em $-0,45 \mathrm{~V}$ (Ic) depende do potencial inicial de varredura. Quando a varredura é iniciada em potenciais menores que $1,2 \mathrm{~V}$, o pico catódico Ic não é registrado. Esses resultados indicam que o composto responsável pelo pico catódico (Ic) é o produto da reação de oxidação da NTAC que permaneceu na dupla camada durante a varredura inversa.

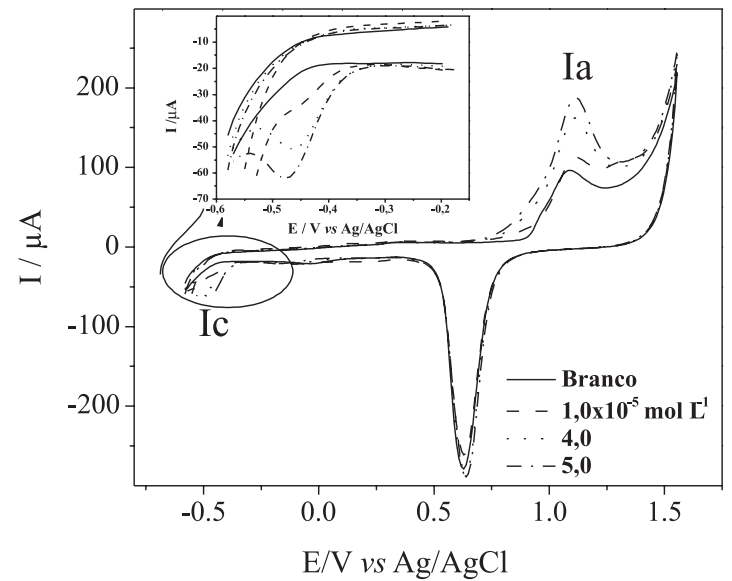

Figura 1. Voltamogramas cíclicos obtidos para o eletrodo de ouro na ausência e na presença de NTAC na concentração de 4,70x10-5 mol L-1 em $\mathrm{Na}_{2} \mathrm{HPO}_{4}$

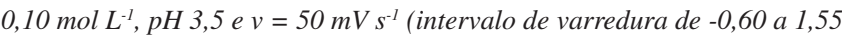
$V)$. Inserção: expansão dos voltamogramas na região de $-0,10$ a $-0,60 \mathrm{~V}$

Para obter maiores informações sobre a influência do potencial inicial de varredura sobre as intensidades de correntes dos picos Ia e Ic foi utilizada a voltametria de onda quadrada com redissolução catódica (CSSWV).

Observa-se na Figura 2 que a intensidade de corrente do pico Ic aumenta em função do potencial aplicado e do tempo de deposição. Para potenciais maiores que $1,0 \mathrm{~V}$ a corrente passa a independer do potencial. Esse comportamento pode ser atribuído ao átomo de enxofre da NTAC, uma vez que o mesmo não foi observado para outras nitrosaminas como $\mathrm{N}$-nitrosodimetilamina (NDMA), N-nitrosodietilamina (NDEA), N-nitropiperidina (NPIP) e N-nitrosopirrolidina (NPIR).

Os resultados observados indicam que o enxofre presente na NTAC é oxidado sobre o eletrodo de DDB em $1,2 \mathrm{~V}$ e o produto formado fica adsorvido na superfície do eletrodo, sendo então redissolvido na varredura reversa em $-0,45 \mathrm{~V}$.

A intensidade de corrente do pico catódico em 1,2 V é dependente da concentração de NTAC, mas uma vez que esse processo redox é sobreposto pela formação de óxido de ouro, não foi obtida uma repetibilidade adequada entre medidas sucessivas para uma mesma 


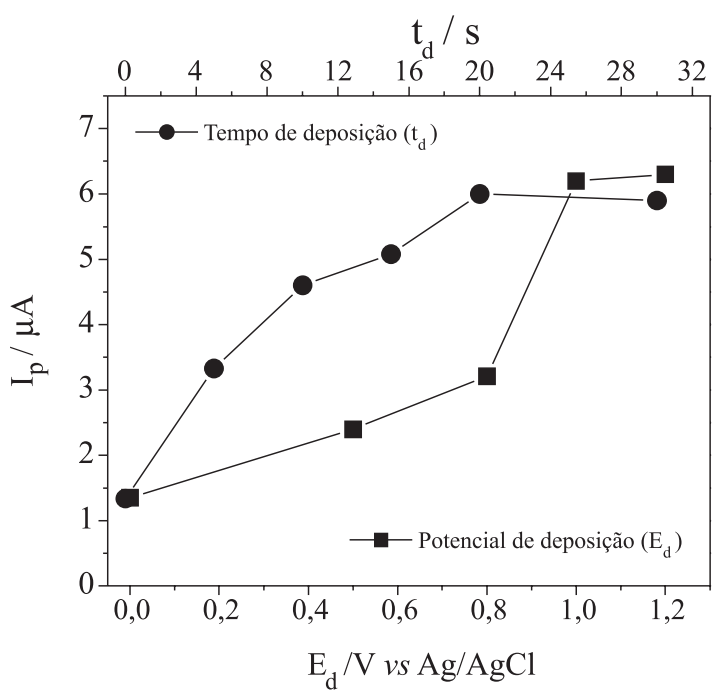

Figura 2. Variação da intensidade da corrente de pico para a NTAC em função do potencial e do tempo de deposição (NTAC 4,70x10 $0^{-5} \mathrm{~mol} \mathrm{~L}^{-1}$ sobre o eletrodo ouro em $\mathrm{Na}_{2} \mathrm{HPO}_{4} 0,10 \mathrm{~mol} \mathrm{~L}^{-1}, \mathrm{pH} \mathrm{3,5,f}=100 \mathrm{~s}^{-1}$, a $=50 \mathrm{mVe}$ $\left.\Delta \mathrm{E}_{\mathrm{s}}=2 \mathrm{mV}\right)$

concentração de NTAC (medidas realizadas na presença de nitrogênio super-seco). Em decorrência deste fato, o sinal em -0,45 V foi o selecionado para fins quantitativos.

Após a obtenção dos valores otimizados do tempo de deposição (20 s) e do potencial de deposição $(1,2 \mathrm{~V})$, todas as medidas voltamétricas foram realizadas utilizando a voltametria de onda quadrada com redissolução catódica (CSSWV).

Os voltamogramas de CSSWV para a NTAC apresentaram um comportamento semelhante àqueles obtidos por voltametria cíclica, ou seja, com somente um pico catódico irreversível em torno de $-0,45 \mathrm{~V}$ vs $\mathrm{Ag} / \mathrm{AgCl}$.

\section{Influência do pH do meio}

O pico de redissolução catódico observado para a NTAC sobre o eletrodo de ouro é fortemente influenciado pelo $\mathrm{pH}$ do meio. Os voltamogramas obtidos para a NTAC em função do pH estão apresentados na Figura 3. A maior intensidade de corrente foi registrada em $\mathrm{pH} 3,5$. Adicionalmente, o potencial de pico sofre variações

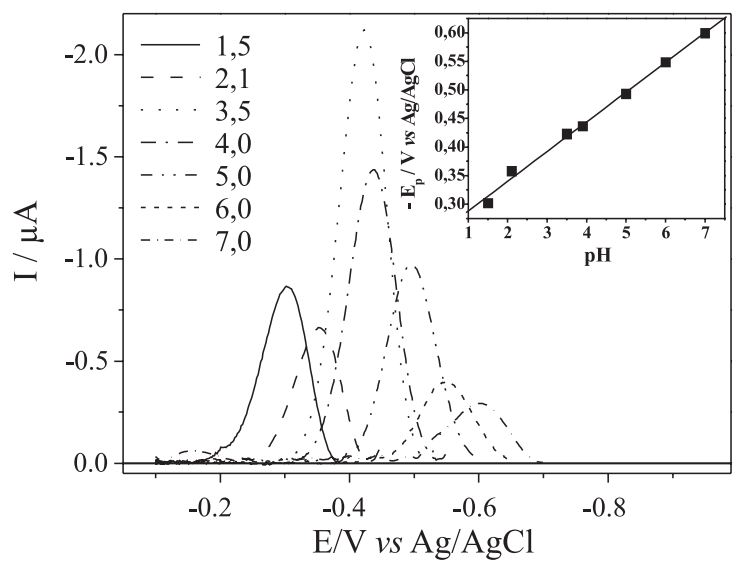

Figura 3. Voltamogramas de onda quadrada com redissolução catódica obtidos para a NTAC $1,00 \times 10^{-5} \mathrm{~mol} \mathrm{L^{-1 }}$ sobre o eletrodo ouro em função do $\mathrm{pH}\left(\mathrm{Na}_{2} \mathrm{HPO}_{4} 0,10 \mathrm{~mol} \mathrm{~L}^{-1}, E_{d}=1,2 \mathrm{~V}, t_{d}=20 \mathrm{~s}, f=100 \mathrm{~s}^{-1}, \mathrm{a}=50 \mathrm{mVe} \Delta \mathrm{E}_{\mathrm{s}}\right.$ $=2 \mathrm{mV}$ ). Inserção: Variação do potencial de pico com o pH para a NTAC com o aumento do $\mathrm{pH}$, provocando o deslocamento do potencial de pico para regiões mais negativas. O coeficiente angular obtido a partir do gráfico $\mathrm{E}_{\mathrm{p}}$ versus $\mathrm{pH}$ (inserção Figura 3) foi de $52 \mathrm{mV} / \mathrm{pH}$ e corresponde a uma transferência de um próton para cada elétron envolvido no processo. ${ }^{19}$

\section{Influência dos parâmetros da SWV para a NTAC sobre o eletrodo de ouro}

A voltametria de onda quadrada é uma das técnicas voltamétricas de pulso mais rápidas e sensíveis. Os limites de detecção podem ser comparados aos das técnicas cromatográficas. Além disso, a análise de parâmetros característicos desta técnica, como a frequiência e a amplitude da onda quadrada e o incremento de varredura, permite a aquisição de informações quanto à cinética e ao mecanismo do processo eletroquímico em questão.

Por meio da variação da freqüência da onda quadrada para a NTAC sobre o eletrodo de ouro, foi observado que um aumento no valor da frequiência causa um aumento proporcional na intensidade de corrente de pico, de acordo com a Equação: $\mathrm{I}_{\mathrm{p}}(\mu \mathrm{A})=0,193+0,020 f\left(\mathrm{~s}^{-1}\right)$. No entanto, frequiências acima de $150 \mathrm{~s}^{-1}$ não influenciam de maneira significativa na intensidade do sinal e por este motivo a frequiência de trabalho escolhida foi a de $150 \mathrm{~s}^{-1}$. De acordo com dados da literatura, ${ }^{20,21}$ a intensidade de corrente para sistemas totalmente irreversíveis com processo controlado pela adsorção das espécies varia linearmente com a freqüência de aplicação dos pulsos, o que corrobora os resultados obtidos para a NTAC sobre o eletrodo de ouro.

Sabendo-se que a espécie é adsorvida na superfície do eletrodo e utilizando-se os critérios de diagnósticos disponíveis para a técnica de voltametria de onda quadrada, foi possível determinar o valor de $\alpha$ n para o sistema. De acordo com a teoria da voltametria de onda quadrada, a dependência dos potenciais de pico com o logaritmo da frequiência para reações totalmente irreversíveis com reagentes e/ou produtos adsorvidos é linear e pode ser descrita pela Equação 1:22

$$
\frac{\Delta E_{p}}{\Delta \log f}=\frac{2,3 R T}{\alpha n F}
$$

onde: $\alpha$ é o coeficiente de transferência de carga e n o número de elétrons envolvidos na reação eletródica.

A variação do potencial de pico em função do logaritmo da freqüência para a NTAC sobre o eletrodo de ouro apresentou um comportamento linear com um coeficiente angular de 0,0501. Mediante a Equação 1 tem-se que $\alpha$ é igual a 1,18. Considerando-se $\alpha$ próximo a 0,5 , estima-se 2 elétrons envolvidos na etapa de transferência de carga por molécula de NTAC.

Para sistemas redox totalmente irreversíveis a intensidade do sinal analítico na voltametria de onda quadrada sofre grande influência com a variação da amplitude da onda, isto porque a largura de meia onda se mantém constante para amplitudes maiores que $50 \mathrm{mV}$.

Na Figura 4 estão apresentados os voltamogramas obtidos para a NTAC em função da variação da amplitude da onda quadrada. Verifica-se que para valores de amplitudes até $60 \mathrm{mV}$ o potencial de pico permanece constante e a corrente de pico é linearmente dependente da amplitude. Para amplitudes maiores que $60 \mathrm{mV}$ o potencial de pico é deslocado para valores menores de potencial. Adicionalmente, amplitudes maiores parecem não atuar de modo significativo na sensibilidade para propósitos analíticos. Em função disto, optou-se em trabalhar com amplitude da onda quadrada de $60 \mathrm{mV}$.

Foi observado que a variação do incremento de varredura no intervalo de 1 a $10 \mathrm{mV}$ não influencia de modo significativo no comportamento da NTAC sobre o eletrodo de ouro. Desta forma, optou-se em trabalhar com um incremento de varredura de $2 \mathrm{mV}$. 


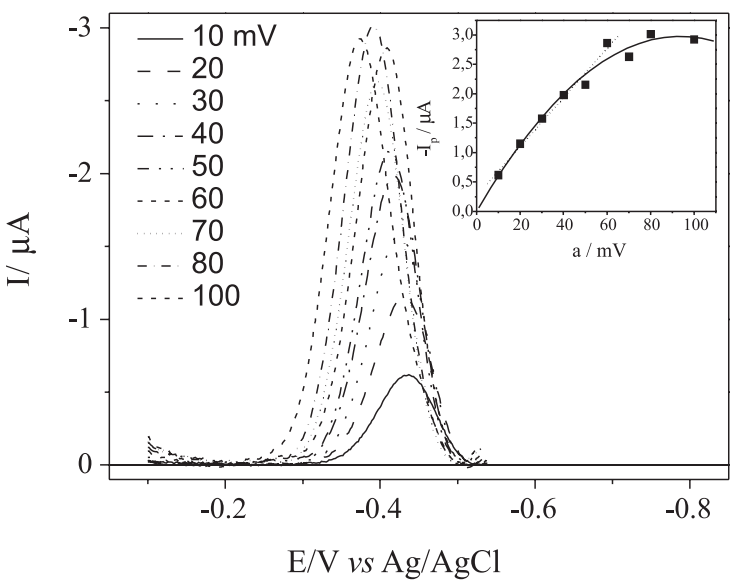

Figura 4. Influência da variação da amplitude da onda quadrada no aspecto geral dos voltamogramas de onda quadrada de redissolução catódica da NTAC $\left(1,20 \times 10^{-5} \mathrm{~mol} \mathrm{~L}^{-1}, \mathrm{pH} 3,5 \mathrm{Na}_{2} \mathrm{HPO}_{4} 0,10 \mathrm{~mol} \mathrm{~L}^{-1}, f=100 \mathrm{~s}^{-1}, \Delta \mathrm{E}_{\mathrm{s}}=2\right.$ $m V, E_{d}=1,2 V, t_{d}=20 \mathrm{~s}$ ). Inserção: Variação da corrente de pico em função da amplitude do pulso para a NTAC sobre o eletrodo de ouro

Variação da corrente de pico em função da concentração de NTAC

Estabelecidas as melhores condições para a determinação de NTAC, através do pico catódico em termos de $\mathrm{pH}(3,5)$, frequiência da onda quadrada $\left(150 \mathrm{~s}^{-1}\right)$, incremento de varredura $(2 \mathrm{mV})$, amplitude da onda quadrada $(60 \mathrm{mV})$, tempo de deposição $(20 \mathrm{~s})$ e potencial de deposição $(1,2 \mathrm{~V})$ foi obtida a curva analítica utilizando-se a CSSWV.

A curva analítica foi construída pela adição de alíquotas da solução padrão de NTAC ao eletrólito suporte composto de $\mathrm{Na}_{2} \mathrm{HPO}_{4} 0,10$ mol L ${ }^{-1}, \mathrm{pH} 3,5$. As medidas foram realizadas em triplicata e para a obtenção da curva analítica foi utilizado o valor médio de corrente de pico para cada nível de concentração. A faixa linear de trabalho foi de $7,0 \times 10^{-8}$ a $2,0 \times 10^{-6} \mathrm{~mol} \mathrm{~L}^{-1}$, com uma linearidade (r) e sensibilidade de 0,9990 e $0,50 \mathrm{~A} \mathrm{~L} \mathrm{~mol}^{-1}$, respectivamente.

Para estabelecer o limite de detecção (LOD), ou seja, a detectabilidade da NTAC na célula eletroquímica, foi utilizada a relação entre o desvio padrão da resposta $\left(\mathrm{S}_{\mathrm{b}}\right)$ e a inclinação da curva analítica (b), ${ }^{23}$ conforme a Equação 2:

$$
L O D=\frac{3 S_{b}}{b}
$$

O limite de detecção obtido para a NTAC foi de $2,1 \times 10^{-8} \mathrm{~mol} \mathrm{~L}^{-1}$ $\left(3,5 \mu \mathrm{g} \mathrm{L}^{-1}\right)$. A precisão intra-ensaio, expressa como a estimativa do desvio padrão relativo, para uma concentração de $1,0 \times 10^{-6} \mathrm{~mol} \mathrm{~L}^{-1}$ ( $\mathrm{n}=10$ ) foi de $2,4 \%$.

Análise da interferência de outras nitrosaminas na determinação de NTAC sobre o eletrodo de ouro

A seletividade do método proposto para a determinação da NTAC foi avaliada quanto à possível interferência de outras nitrosaminas, utilizando-se o eletrodo de ouro e a SWV. As nitroaminas utilizadas para esse estudo foram N-nitroso-dimetilamina (NDMA), N-nitrosodietilamina (NDEA), N-nitropiperidina (NPIP) e N-nitrosopirrolidina (NPIR), uma vez que essas nitrosaminas são freqüentemente encontradas em produtos cárneos curados.

Os resultados obtidos com NDMA, NDEA, NPIP e NPIR mostraram que estas nitrosaminas não são eletroativas no eletrodo de ouro nas condições utilizadas para a determinação eletroanalítica da NTAC. Na Figura 5 é apresentado o comportamento voltamétrico da NTAC, na concentração de $0,2 \times 10^{-6} \mathrm{~mol} \mathrm{~L}^{-1}$, em função da

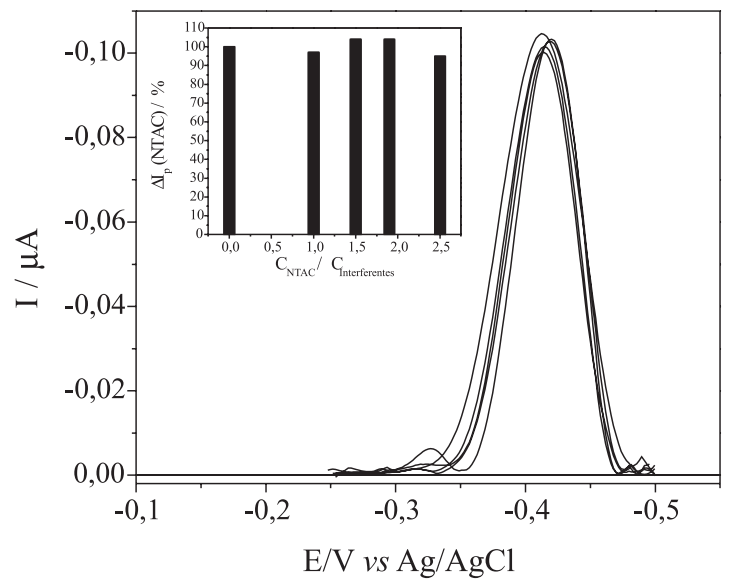

Figura 5. Voltamogramas de onda quadrada de redissolução catódica obtidos

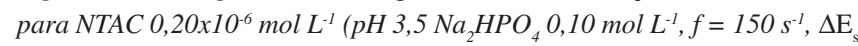
$\left.=2 \mathrm{mV}, a=60 \mathrm{mV}, E_{d}=1,2 \mathrm{~V}, t_{d}=20 \mathrm{~s}\right)$ na presença de outras nitrosaminas. Inserção: Variação da corrente de pico da NTAC em função da adição de NDMA, NDEA, NPIP e NPIR

adição de NDMA, NDEA, NPIP e NPIR. Pode-se observar que não ocorrem mudanças significativas no perfil dos voltamogramas da NTAC e que a corrente de pico se mantém praticamente constante com uma perda de sinal menor que 5\% (inserção da Figura 5). Para uma concentração de NTAC de $1,0 \times 10^{-6} \mathrm{~mol} \mathrm{~L}^{-1}$ resultados semelhantes foram observados, indicando que a NTAC pode ser determinada na presença de outras nitrosaminas, utilizando-se a metodologia proposta.

Os resultados obtidos indicam que a CSSWV, usando o eletrodo de ouro, apresenta detectabilidade, precisão e seletividade adequados para ser empregada na determinação de NTAC em alimentos.

\section{Estudo do comportamento eletroquímico de NTAC sobre o eletrodo de mercúrio}

O estudo do comportamento eletroquímico da NTAC sobre o eletrodo de gota pendente de mercúrio (HMDE) foi realizado para fins comparativos com o processo redox obtido sobre o eletrodo de ouro.

Na Figura 6 é apresentado um voltamograma cíclico da NTAC sobre o HMDE, sendo que apenas é registrado um pico catódico em torno de $-0,99 \mathrm{~V}$ com características irreversíveis.

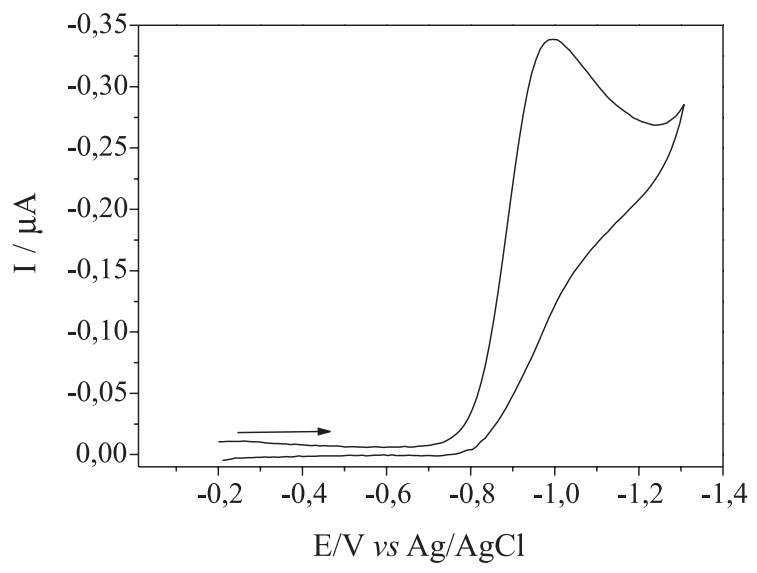

Figura 6. Voltamograma cíclico obtido para a NTAC na concentração de $1,60 \times 10^{-4} \mathrm{~mol} \mathrm{~L}^{-1}$ sobre o eletrodo de mercúrio (HMDE) em $\mathrm{Na}_{2} \mathrm{HPO}_{4} 0,10$ $m o l L^{-1}, p H=3,5$ ev $=50 \mathrm{mV} \mathrm{s}^{-1}$ 
Com o objetivo de se obter informações adicionais sobre o processo redox da NTAC no eletrodo HMDE, foram também realizados estudos dos parâmetros da voltametria de onda quadrada, como a freqüência da onda quadrada $(f)$, a amplitude da onda quadrada $(a)$ e o incremento de varredura $\left(\Delta \mathrm{E}_{\mathrm{s}}\right)$.

Avaliação dos parâmetros da SWV para a NTAC sobre o HMDE

Na Figura 7 é apresentado o comportamento da NTAC sobre o eletrodo de HMDE em função da variação da frequiência da onda quadrada. Observa-se um aumento na intensidade da corrente de pico para frequiências até $300 \mathrm{~s}^{-1}$. Adicionalmente, pode-se verificar (inserção da Figura 7) que a relação da intensidade da corrente de pico é linear com a raiz quadrada da freqüência, o que corrobora com um processo controlado pela difusão das espécies. Estes resultados estão de acordo com os dados da literatura para outras nitrosaminas, onde é observada a redução do grupo $\mathrm{N}$-nitroso para hidroxilamina. ${ }^{13}$

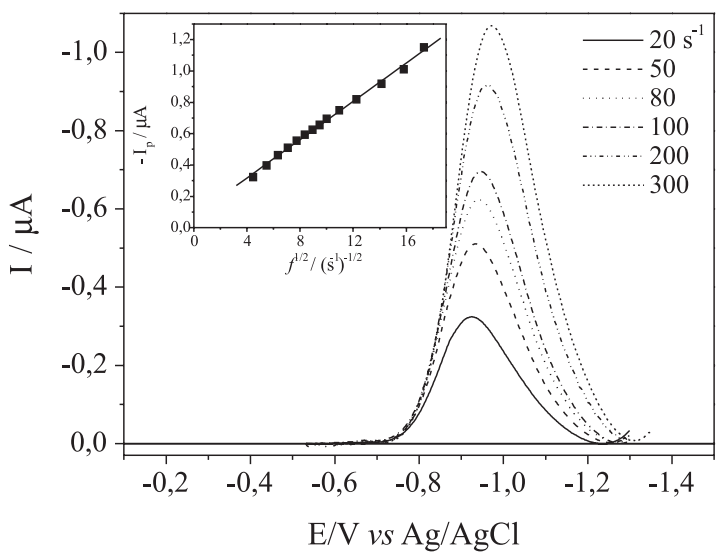

Figura 7. Voltamogramas de onda quadrada obtidos para a NTAC sobre o eletrodo de mercúrio em função da variação da freqüência da onda quadrada $\left(1,60 \times 10^{-4} \mathrm{~mol} \mathrm{~L}^{-1}\right.$ em $\mathrm{Na}_{2} \mathrm{HPO}_{4} 0,10 \mathrm{~mol} \mathrm{~L}^{-1}, \mathrm{pH}=3,5, \mathrm{a}=50 \mathrm{mV}$, e incremento de varredura de $2 \mathrm{mV}$ ). Inserção: Variação da intensidade da corrente de pico com a raiz quadrada da freqüência de aplicação dos pulsos

A amplitude da onda quadrada foi outro parâmetro avaliado e pode-se observar que para valores superiores a $50 \mathrm{mV}$, a amplitude não influencia de maneira significativa na intensidade do sinal, o que está de acordo com o previsto para sistemas totalmente irreversíveis.

Para o incremento de varredura $\left(\Delta \mathrm{E}_{\mathrm{s}}\right)$ foi verificado que ocorre um aumento da intensidade da corrente catódica em função do aumento de $\Delta \mathrm{E}_{\mathrm{s}}$ e o potencial de pico é deslocado para valores mais negativos. Para desenvolvimento da metodologia analítica foi selecionado o valor de $\Delta \mathrm{E}_{\mathrm{s}}$ de $4 \mathrm{mV}$.

Variação da corrente de pico em função da concentração de NTAC $e$ análise da interferência de outras nitrosaminas

Estabelecidas as melhores condições voltamétricas para a determinação da NTAC sobre o eletrodo de mercúrio, em termos de freqüência da onda quadrada $\left(300 \mathrm{~s}^{-1}\right)$, incremento de varredura (2 $\mathrm{mV})$ e amplitude da onda quadrada $(50 \mathrm{mV})$, foi construída a curva

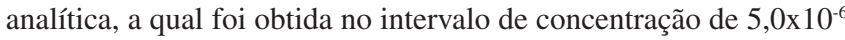
a $5,0 \times 10^{-5} \mathrm{~mol} \mathrm{~L}^{-1}$. Neste intervalo de concentração (faixa linear de trabalho) obteve-se uma sensibilidade de $0,031 \mathrm{~A} \mathrm{~L} \mathrm{~mol}^{-1}$ e linearidade (r) de 0,9970.

A avaliação do sinal analítico obtido para a NTAC sobre o eletrodo de mercúrio, na presença de outras nitrosaminas foi realizada e os resultados obtidos encontram-se na Figura 8. Observa-se que ocorre um aumento na corrente de pico da NTAC quando NDMA,

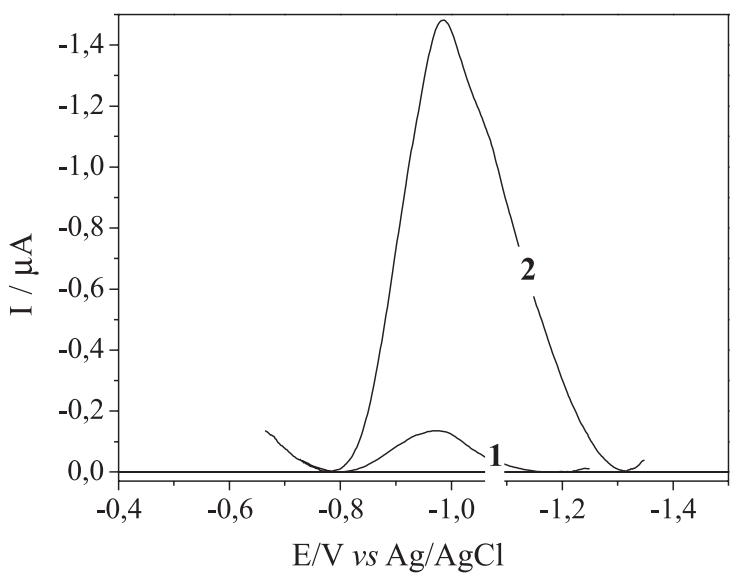

Figura 8. Voltamogramas de onda quadrada obtidos para a NTAC 5,00 $10^{-5}$ mol $L^{-1}$ na ausência (1) e na presença de outras nitrosaminas (NDMA, NDEA, NPIP e NPIR) 5,0 $10^{-5} \mathrm{~mol} \mathrm{~L}^{-1}$ (2) em $\mathrm{Na}_{2} \mathrm{HPO}_{4} 0,10 \mathrm{~mol} \mathrm{~L}^{-1}, \mathrm{pH}=3,5$, a = $50 \mathrm{mV}, f=300 \mathrm{~s}^{-1} e \Delta \mathrm{E}_{\mathrm{s}}=4 \mathrm{mV}$

NDEA, NPIP e NPIR são adicionadas à célula eletroquímica, o que se deve ao fato do sinal observado ser referente à redução do grupo $\mathrm{N}$-nitroso, comum a todas as nitrosaminas.

Desta forma, sobre o eletrodo de mercúrio não foi possível a determinação seletiva da NTAC, sendo possível somente a quantificação de nitrosaminas totais nas condições estudadas. Estes resultados confirmam que o sinal observado para a NTAC sobre o eletrodo de mercúrio é proveniente da redução do grupo N-nitroso e é diferente do processo redox observado no eletrodo de ouro.

\section{Comparação dos resultados obtidos para a NTAC sobre os eletrodos de ouro e mercúrio}

Na Tabela 1 estão reunidos os principais resultados obtidos para NTAC sobre os eletrodos de ouro e HMDE, os quais indicam a evidente diferença do comportamento eletroquímico desta nitrosamina sobre as duas superfícies eletródicas estudadas.

Tabela 1. Parâmetros voltamétricos obtidos para a NTAC sobre os eletrodos de ouro e HMDE

\begin{tabular}{|c|c|c|}
\hline Parâmetro & Eletrodo de Ouro & HMDE \\
\hline Intervalo de potencial (V) & $-0,60$ a 1,55 & $-0,20 \mathrm{a}-1,40$ \\
\hline Picos anódicos $(\mathrm{V})$ & 1,20 & - \\
\hline Picos catódicos (V) & $-0,41$ & $-0,99$ \\
\hline Técnica voltamétrica & CSSWV & SWV \\
\hline $\begin{array}{l}\text { Características do } \\
\text { processo eletroquímico }\end{array}$ & $\begin{array}{l}\text { Irreversível } \\
\text { controlado } \\
\text { por adsorção }\end{array}$ & $\begin{array}{l}\text { Irreversível } \\
\text { controlado } \\
\text { por difusão }\end{array}$ \\
\hline $\begin{array}{l}\text { Intervalo linear de } \\
\text { concentração }\left(\mathrm{mol} \mathrm{L}^{-1}\right)\end{array}$ & $7,0 \times 10^{-8}$ a $2,0 \times 10^{-6}$ & $5,0 \times 10^{-6}$ a $5,0 \times 10^{-5}$ \\
\hline $\begin{array}{l}\text { Seletividade na presença } \\
\text { de outras nitrosaminas* }\end{array}$ & Seletivo & Não seletivo \\
\hline
\end{tabular}

* NDMA, NDEA, NPIR e NPYR

Sobre o eletrodo HMDE os resultados demonstram que o sinal observado é proveniente da redução do grupo N-nitroso, uma vez que na presença de outras nitrosaminas houve uma sobreposição dos sinais e não foi possível fazer o estudo seletivo da NTAC nas condições estudadas. Adicionalmente, dados da literatura relatados para outras nitrosaminas sobre o eletrodo de mercúrio estão de acor- 
do com o observado para a NTAC e com o mecanismo de redução apresentado no Esquema 1.

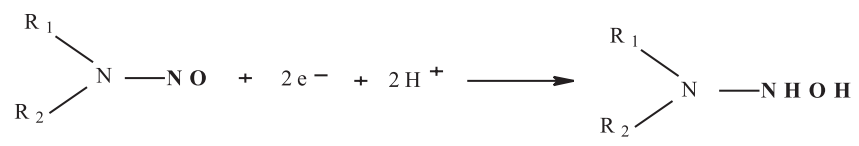

Esquema 1. Proposta de mecanismo de redução da NTAC sobre o eletrodo de mercúrio

A resposta voltamétrica da NTAC sobre o eletrodo de ouro depende da oxidação do enxofre, o que está de acordo com dados da literatura, ${ }^{24}$ que mostram que moléculas contendo enxofre são facilmente oxidadas sobre eletrodos de ouro. O produto de oxidação da NTAC fica adsorvido na superfície do ouro e sofre redução na varredura catódica em $-0,45 \mathrm{~V}$, mediante transferência de dois elétrons. $\mathrm{O}$ processo é próton dependente.

\section{CONCLUSÕES}

Foi observado que o processo redox da NTAC no eletrodo de ouro e no de mercúrio (HMDE) seguem mecanismos diferenciados. Enquanto no eletrodo de mercúrio ocorre a redução do grupo Nnitroso, no eletrodo de ouro a oxidação do enxofre é responsável pela eletroatividade do composto. O produto de oxidação da NTAC sofre adsorção na superfície do eletrodo de ouro e na varredura catódica ocorre redução desta espécie, mediante transferência de dois elétrons, sendo que a corrente de pico é proporcional à concentração de NTAC na célula voltamétrica. Essa diferença no comportamento eletroquímico da NTAC nos dois materiais eletródicos é importante do ponto de vista analítico, pois permite a determinação seletiva da NTAC na presença de outras nitrosaminas, usando o eletrodo de ouro, fato esse que permitiu o desenvolvimento de um método, empregando a voltametria de onda quadrada de redissolução catódica.

O método voltamétrico desenvolvido apresentou detectabilidade e seletividade adequada para a determinação direta de NTAC, assim como, o eletrodo de ouro nestas condições, apresenta potencialidade para ser utilizado como detetor eletroquímico associado a sistemas de cromatografia líquida de alta eficiência para análise de alimentos.

\section{AGRADECIMENTOS}

À FAPESP e ao CNPq pelo suporte financeiro. L. Codognoto agradece à FAPESP pela bolsa de estudos recebida (Proc. 03/13547-6).

\section{REFERÊNCIAS}

1. Douglass, M. L.; Kabacoff, B. L.; Anderson, G. A.; Cheng, M. C.; J. Soc. Cosmet. Chem. 1978, 29, 581.

2. Gray, J. I.; Randall, C. J.; J. Food Protection 1979, 42, 168.

3. Institute of Food Technologists - Scientific Status Summary; Food Technol. 1987, 41, 127.

4. Hotchkiss, J. A.; Addit. Food Res. 1987, 31, 54.

5. Mirvish, S. S.; Toxic. Appl. Pharm. 1975, 31, 325.

6. Walters, C. L.; Food Addit. Contam. 1992, 9, 441.

7. Pensabene, J. W.; Fiddler, W.; J. AOAC Int. 1994, 77, 981.

8. Raoul, S.; Gremaud, E.; Biaudet H.; Turesky R. J.; J. Agric. Food Chem. 1997, 45, 4706 .

9. Andrade, R.; Reyes, F. G. R.; Rath, S.; Food Chem. 2005, 91, 173.

10. Zhao, Y. Y.; Boyd, J.; Hrudey, S. E.; Li, X.; Environm. Sci. Technol. 2006, 40, 763.

11. Munch, J. W.; Bassett, M. V.; J. AOAC Int. 2006, 89, 496.

12. Goicolea, M. A.; Balugera, Z. G.; Portela, M. J.; Barrio, R. J.; Anal. Chim. Acta 1995, 305, 310.

13. Belal, F.; Walash, M. I.; Ibrahim, F.; Hefnawy, M.; Eid, M.; Il Fármaco 2000, 55, 694.

14. Gorski, W.; Cox, J. A.; Anal. Chem. 1994, 66, 2771.

15. Gorski, W.; Cox, J. A.; J. Electroanal. Chem. 1995, 389, 123.

16. Cox, J. A.; Alberr, K. S.; Brockway, C. A.; Tess, M. E.; Gorski, W.; Anal. Chem. 1995, 67, 993.

17. Collyer, S. D.; Bradbury, S.; Hatfield, J. V.; Higson, S. P. J.; Electroanalysis 2001, 13, 332.

18. Collyer, S. D.; Buther, A. J.; Higson, S. P. J.; Electroanalysis 1997, 9 , 985.

19. Bard, A. J.; Faulkner, L. R.; Electrochemical Methods: Fundaments and Applications, John Wiley: New York, 2001.

20. Osteryoung, J. G.; O’Dea, J. J. Em Electroanalytical Chemistry; Bard, A. J., ed.; Marcel Dekker: New York, 1982, vol. 14, p. 459.

21. Souza, D.; Machado, S. A. S.; Avaca, L. A.; Quim. Nova 2003, $26,81$.

22. Lovric, M.; Komorsky-Lovric, S.; J. Electroanal. Chem. 1988, 248, 239.

23. Long, G. L.; Winefordner, J. D.; Anal. Chem. 1983, 55, 712A.

24. Svensmark, B. O.; Em Organic Electrochemistry; Marcel Dekker: New York, 1991, vol. 3, p. 659. 